\title{
Probing Entanglement and Non-locality of Electrons in a Double-Dot via Transport and Noise
}

\author{
Daniel Loss and Eugene V. Sukhorukov \\ Department of Physics and Astronomy, University of Basel, \\ Klingelbergstrasse 82, CH-4056 Basel, Switzerland
}

(October 24, 2018)

\begin{abstract}
Addressing the feasibilty of quantum communication with electrons we consider entangled spin states of electrons in a double-dot which is weakly coupled to in-and outgoing leads. We show that the entanglement of two electrons in the double-dot can be detected in mesoscopic transport and noise measurements. In the Coulomb blockade and cotunneling regime the singlet and triplet states lead to phase-coherent current and noise contributions of opposite signs and to Aharonov-Bohm and Berry phase oscillations in response to magnetic fields. These oscillations are a genuine two-particle effect and provide a direct measure of non-locality in entangled states. We show that the ratio of zero-frequency noise to current (Fano factor) is universal and equal to the electron charge.
\end{abstract}

Entanglement and non-locality of Einstein-PodolskyRosen (EPR) pairs [1] are remarkable features of quantum mechanics which give rise to striking phenomena such as violation of Bell inequalities and secure quantum communication [2]. Such and related phenomena have been tested with great success for photons [3, 14, but not yet for massive particles such as electrons, particularly in a solid state environment. There are two immediate problems. First we need a scheme by which entanglement of electrons can be generated in a controlable manner. In the context of quantum computing [5] we have recently shown that such a scheme can be realized in tunnel-coupled quantum dots each of which contains one single (excess) electron whose spin defines the qubit [6 8]. There are several motivations for such a qubit scheme, most notably very long spin decoherence times [9], and the qubit defined as electron-spin is mobile and thus is a good candidate for implementing quantum communication schemes which are based on EPR pairs 10. An important feature of this scheme, moreover, is that we have control over the non-locality of the entangled state: electron 1 and 2 are localized in different quantum dots and while being spatially separated from each other their total spin ground state (a singlet) is entangled [11].

The second problem which then immediately arises is: How can we probe entanglement? In the following we provide an answer to this question and show that this property can be tested via mesoscopic transport and noise measurements. The scheme we propose consists of two coupled quantum dots - double-dot (DD) for shortwhich themselves are weakly coupled to two leads 1 and 2 (see Fig. 1). In contrast to earlier set-ups involving a DD [12,13], we propose a scheme where an electron coming from lead 1 (2) has the option to tunnel into both dot 1 and dot 2. This results in a closed loop, and applying a magnetic field, an Aharonov-Bohm (AB) phase $\varphi$ will be accumulated by an electron traversing the DD. In the Coulomb blockade $(\mathrm{CB})$ regime we find that due to

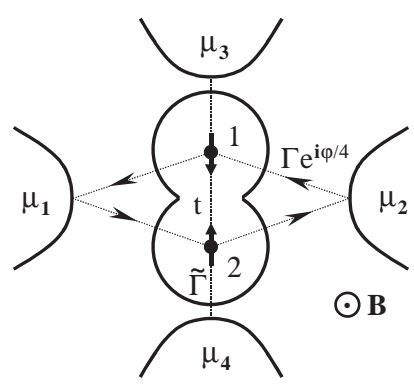

a)

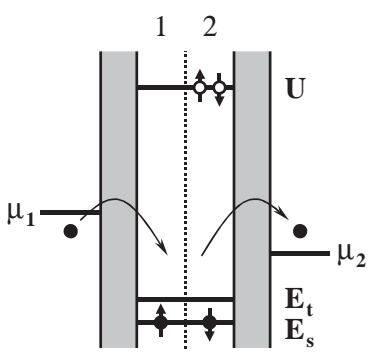

b)
FIG. 1. a) Double-dot (DD) system containing two electrons and being weakly coupled to metallic leads $1, \ldots, 4$, each of which is at the chemical potential $\mu_{1}, \ldots, \mu_{4}$. The tunneling amplitudes between dots and leads are denoted by $\Gamma, \tilde{\Gamma}$. The tunneling ( $\mathrm{t}$ ) between the dots results in a singlet ground state [7] with energy $E_{s}$ and a triplet state with $E_{t}$, with $J=E_{t}-E_{s}$ describing the effective exchange coupling between the electron spins (qubits). The closed tunneling path between dots and leads 1 and 2 encloses the area $A$. b) Energy diagram of the DD system described in a) in the CB regime where cotunneling is dominant, i.e., $U>\left|\mu_{1} \pm \mu_{2}\right|>J$, with $U$ being the Coulomb repulsion within a single dot.

cotunneling [14] the current and the noise depend on the state of the $\mathrm{DD}$ : the $\mathrm{AB}$ oscillations for singlet and triplets have opposite sign. The amplitude of the $\mathrm{AB}$ oscillations provides a measure of the phase coherence of the entangled state, while the period -via the enclosed area-provides a measure of the non-locality of the EPR pairs. The triplets themselves can be further distinguished by applying a directionally inhomogeneous magnetic field which adds a Berry phase leading to beating. Finally, we also discuss finite frequency noise, and show in particular that the power spectrum for a DD contains a non-vanshing odd-frequency part which is sensitive to entanglement.

Model System. The DD system (see Fig. 1) contains 
4 metallic leads which are in equilibrium with associated reservoirs kept at the chemical potentials $\mu_{i}, i=1, \ldots, 4$, where the currents $I_{i}$ can be measured. The leads are weakly coupled to the dots with amplitudes $\Gamma$ and $\tilde{\Gamma}$, and the leads 1,2 are coupled to both dots and play the role of probes where the current and noise are measured. The leads 3 and 4 are feeding electrodes to manipulate the electron filling in the dots. The quantum dots contain one (excess) electron each, and are coupled to each other by the tunneling amplitude $t$, which leads to a level splitting [6,7] $J=E_{t}-E_{s} \sim 4 t^{2} / U$ of the single-particle energy levels in the $\mathrm{DD}$, with $U$ being the single-dot Coulomb repulsion energy, and $E_{s / t}$ are the singlet/triplet energies [15. We recall that for two electrons in the DD (and for weak magnetic fields) the ground state is given by a spin singlet [6, 7]. For convenience we count the chemical potentials $\mu_{i}$ from $E_{s}$. The coupling $\tilde{\Gamma}$ to the feeding leads can be be switched off while probing the DD with a current. From now on we assume that $\tilde{\Gamma}=0$ unless stated otherwise.

Using a standard tunneling Hamiltonian approach [16], we write $H=H_{0}+V$, where the first term in $H_{0}=H_{D}+$ $H_{1}+H_{2}$ describes the DD and $H_{1,2}$ the leads (assumed to be Fermi liquids). The tunneling between leads and dots is described by the perturbation $V=V_{1}+V_{2}$, where

$$
\begin{aligned}
& V_{n}=\Gamma \sum_{s}\left[D_{n, s}^{\dagger} c_{n, s}+c_{n, s}^{\dagger} D_{n, s}\right], \\
& D_{n, s}=e^{ \pm i \varphi / 4} d_{1, s}+e^{\mp i \varphi / 4} d_{2, s}, \quad n=1,2,
\end{aligned}
$$

and where the operators $c_{n, s}$ and $d_{n, s}$ annihilate electrons with spin $s$ in the $n$th lead and in the $n$th dot, resp. The Peierls phase $\varphi$ in the hopping amplitude accounts for an $\mathrm{AB}$ or Berry phase (see below) in the presence of a magnetic field. The upper sign belongs to lead 1 and the lower to lead 2. Finally we assume that spin is conserved in the tunneling process. For the outgoing currents we have $I_{n}=i e \Gamma \sum_{s}\left[D_{n, s}^{\dagger} c_{n, s}-c_{n, s}^{\dagger} D_{n, s}\right]$. The observables of interest are the average current through the DD system, $I=\left\langle I_{2}\right\rangle$, and the symmetrized cross correlations (noise) of the outgoing currents, $S(t)=\operatorname{Re}\left\langle\delta I_{2}(t) \delta I_{1}(0)\right\rangle$, where $\delta I_{2}=I_{2}-I$ etc. First, we evaluate the transport current and then the noise.

Cotunneling current. From now on we concentrate on the $\mathrm{CB}$ regime where we can neglect double (or more) occupancy in each dot for all transitions including virtual ones, i.e. we require $\mu_{1,2}<U$. Further we assume that $\mu_{1,2}>J, k_{B} T$ to avoid resonances which might change the DD state (see also below). The lead-dot coupling $\Gamma$ is assumed to be weak so that the state of the DD is not perturbed; this will allow us to retain only the first nonvanishing contribution in $\Gamma$ to $I$ and $S(t)$. Formally, we require $J>2 \pi \nu_{t} \Gamma^{2}$, where $\nu_{t}$ is the tunneling density of states of the leads (see below).

In analogy to the single-dot case [14,17, we refer to above $\mathrm{CB}$ regime as cotunneling regime. In this regime electrons tunnel one by one through virtual states of the DD (see Fig.1). For real quantum dots not all of our assumptions might be perfectly satisfied and phase coherence might be supressed. Still, since it will turn out that in the cotunneling regime the current has a phase-coherent part with $\mathrm{AB}$ oscillations, it should be possible to extract this part even if its amplitude is much smaller than the incoherent one (this is a common situation in mesoscopic transport experiments) [19]. Thus, from now on we will concentrate on cotunneling only. We have specified now all assumptions [18] under which the following results are valid, again they are $U>\mu_{1,2}>k_{B} T, J>2 \pi \nu_{t} \Gamma^{2}$.

Continuing with our derivation of $I$, we note that the average $\langle\ldots\rangle \equiv \operatorname{Tr} \rho\{\ldots\}$ is taken with respect to the equilibrium state of the entire system set up in the distant past before $V$ is switched on [16]. Then, in the interaction picture, the current is given by

$$
I=\left\langle U^{\dagger} I_{2}(t) U\right\rangle, \quad U=T \exp \left[-i \int_{-\infty}^{t} d t^{\prime} V\left(t^{\prime}\right)\right]
$$

The leading contribution in $\Gamma$ to the cotunneling current involves the tunneling of one electron from the DD to, say, lead 2 and of a second electron from lead 1 to the DD (see Fig.1). This contribution is of order $V_{2} V_{1}^{2}$, and thus $I \propto \Gamma^{4}$, as is typical for cotunneling [14].

After some manipulations we can express the current (2) in terms of spinless Green functions of the leads evaluated at coinciding points, $G_{i}^{>}(t)=-i\left\langle c_{i}(t) c_{i}^{\dagger}(0)\right\rangle$ and $G_{i}^{<}(t)=i\left\langle c_{i}^{\dagger}(0) c_{i}(t)\right\rangle$ 16. The expanded expression for $I$ is too lengthy to be presented here, so we only mention that all terms of the form $G_{1}^{>} G_{2}^{>}$contain divergencies at small energy transfer around the DD levels due to resonances [23]. This is seen from the structure of $G_{i}^{>}$: first an electron is created in the leads and then annihilated, thus describing a resonant current away from the DD into leads 1 and 2. If the state in the DD is maintained through the coupling to feeding leads 3 and 4 [23], these resonances will be smeared and the divergencies have to be cut at an energy $\varepsilon \sim 2 \pi \nu_{t} \tilde{\Gamma}^{2}$. On the other hand, in the cotunneling regime defined above we are far away from such resonances, and such a resonant current is suppressed, and all divergent terms of the form $G_{1}^{>} G_{2}^{>}$vanish. We arrive then at the following compact expression for the cotunneling current

$$
\begin{aligned}
& I=\frac{e \Gamma^{4}}{2 \pi} \sum_{i, f, s, s^{\prime}} \rho_{i}\left|\left\langle i\left|D_{2, s^{\prime}}^{\dagger} D_{1, s}\right| f\right\rangle\right|^{2}\left[F_{12}-F_{21}\right], \\
& F_{12}=\int \frac{d \varepsilon}{\varepsilon^{2}} G_{1}^{<}\left(\varepsilon+E_{f}\right) G_{2}^{>}\left(\varepsilon+E_{i}\right) .
\end{aligned}
$$

For simplicity, we assumed that the upper and lower branches of the tunneling loop (Fig.1) are identical. Eqs. (3) and (4) show that in the cotunneling regime the initial state $|i\rangle$ (with weight $\rho_{i}$ and energy $E_{i}$ ) of the DD is changed into a final state $|f\rangle$ (energy $E_{f}$ ) by the traversing electron. However, due to the weak coupling $\Gamma$, the DD will have returned to its equilibrium state before 
the next electron passes through it [18. For small bias, $\left|\mu_{1}-\mu_{2}\right|<J$, only elastic cotunneling is allowed [22], i.e. $E_{i}=E_{f}$. However, this regime is not of interest here since singlet and triplet contributions turn out to be identical and thus indistinguishable 23. We thus focus on the opposite regime, $\left|\mu_{1}-\mu_{2}\right|>J$, where inelastic cotunneling 24 occurs with singlet and triplet contributions being different. In this regime we can neglect the dynamics generated by $J$ compared to the one generated by the bias ("slow spins"), and drop the energies $E_{i}$ and $E_{f}$ in the Eq. (4). Finally, using $1=\sum_{f}|f\rangle\langle f|$ we obtain

$$
\begin{aligned}
& I=e \pi \nu_{t}^{2} \Gamma^{4} C(\varphi) \frac{\mu_{1}-\mu_{2}}{\mu_{1} \mu_{2}} \\
& C(\varphi)=\sum_{s, s^{\prime}}\left[\left\langle d_{1 s^{\prime}}^{\dagger} d_{1 s} d_{1 s}^{\dagger} d_{1 s^{\prime}}\right\rangle+\cos \varphi\left\langle d_{1 s^{\prime}}^{\dagger} d_{1 s} d_{2 s}^{\dagger} d_{2 s^{\prime}}\right\rangle\right]
\end{aligned}
$$

where we assumed Fermi liquid leads, $G_{n}^{>}(\varepsilon)=$ $-i \pi \nu_{t} f_{F}\left(\mu_{n}-\varepsilon\right)$, and $G_{n}^{<}(\varepsilon)=i \pi \nu_{t} f_{F}\left(\varepsilon-\mu_{n}\right)$, where the tunneling density of states in the leads $\nu_{t}=$ $-\frac{2}{\pi} \operatorname{Im} \sum_{p} G^{R}(\varepsilon, p)$ is expressed in terms of the retarded Green function [16], and $f_{F}$ is the Fermi function.

Eq. (5) shows that the cotunneling current depends on the properties of the equilibrium state of the DD through the coherence factor $C(\varphi)$ given in (6). The first term in $C$ is the contribution from the topologically trivial tunneling path (phase-incoherent part) which runs from lead 1 through, say, dot 1 to lead 2 and back. The second term (phase-coherent part) in $C$ results from an exchange process of electron 1 with electron 2 via the leads 1 and 2 such that a closed loop is formed enclosing an area $A$ (see Fig. 1). Note that for singlet and triplets the initial and final spin states are the same after such an exchange process. Thus, in the presence of a magnetic field $B$, an AB phase factor $\varphi=A B e / h$ is acquired.

Next, we evaluate $C(\varphi)$ explicitly in the singlet-triplet basis: $|S\rangle=\frac{1}{\sqrt{2}}(|\uparrow \downarrow\rangle-|\downarrow \uparrow\rangle),\left|T_{0}\right\rangle=\frac{1}{\sqrt{2}}(|\uparrow \downarrow\rangle+|\downarrow \uparrow\rangle)$, $\left|T_{+}\right\rangle=|\uparrow \uparrow\rangle$, and $\left|T_{-}\right\rangle=|\downarrow \downarrow\rangle$. Note that only the singlet $|S\rangle$ and the triplet $\left|T_{0}\right\rangle$ are entangled EPR pairs while the remaining triplets are not (they factorize). Assuming that the DD is in one of these states we obtain the important result

$$
C(\varphi)=2 \mp \cos \varphi \text {. }
$$

Thus, we see that the singlet (upper sign) and the triplets (lower sign) contribute with opposite sign to the phasecoherent part of the current. One has to distinguish, however, carefully the entangled from the non-entangled states. The phase-coherent part of the entangled states is a genuine two-particle effect, while the one of the product states cannot be distinguished from a phase-coherent single-particle effect [19]. Indeed, this follows from the observation that the phase-coherent part in $C$ factorizes for the product states $T_{ \pm}$while it does not so for $S, T_{0}$. Also, for states such as $|\uparrow \downarrow\rangle$ the coherent part of $C$ vanishes, showing that two different (and fixed) spin states cannot lead to a phase-coherent contribution since we know which electron goes which part of the loop.

Finally we note that due to the $\mathrm{AB}$ phase the role of the singlet and triplets can be interchanged which is to say that we can continually transmutate the statistics of the entangled pairs $S, T_{0}$ from fermionic to bosonic (like in anyons): the symmetric orbital wave function of the singlet $S$ goes into an antisymmetric one at half a flux quantum, and vice versa for the triplet $T_{0}$.

Next, we allow for equal population of the singlet and triplet states in the DD, $\rho_{i}=1 / 4, i=1, \ldots, 4$. Experimentally, this can be achieved e.g. by raising the tunnel barrier between the dots (or by increasing the magnetic field) [6.7] such that $J$ vanishes or just becomes smaller than $k_{B} T$. Tracing then over the 4 terms we see that the EPR pairs, $S, T_{0}$, cancel each other while the unentangled terms, $T_{ \pm}$, add up. [This tracing can also be performed over the standard product spin basis as typically used for Fermi liquids.] Thus, in this case we return to the usual Fermi sea situation where we can no longer distinguish single- from two-particle phase-coherence [25]. We note that this effect can be exploited to search for entanglement: first prepare the DD in its entangled ground state (singlet) with $\left|\mu_{1} \pm \mu_{2}\right|>J>k_{B} T$, and then reduce $J$ below $k_{B} T$; after some spin relaxation time an equal population of singlet and triplet states (all contributing with weight $1 / 4$ ) is reached with a concommitant increase of the current by a factor of $5 / 2$.

We would like to stress that the amplitude of the $A B$ oscillations is a direct measure of the phase coherence of the entanglement, while the period via the enclosed area $A=h / e B_{0}$ gives a direct measure of the non-locality of the EPR pairs, with $B_{0}$ being the field at which $\varphi=1$. The triplets themselves can be further distinguished by applying a directionally inhomogeneous magnetic field (around the loop) producing a Berry phase $\Phi^{B}$ [26], which is positive (negative) for the triplet $m=1(-1)$, while it vanishes for the EPR pairs $S, T_{0}$. Thus, for $J=0$ we will eventually see beating in the $\mathrm{AB}$ oscillations due to the positive (negative) shift of the AB phase $\Phi$ by the Berry phase, $\varphi=\Phi \pm \Phi^{B}$. We finally note that the closed loop shown in Fig.1 can actually be made as large as the dephasing length by replacing the dotted lines outside the dots e.g. by wave guides forming a loop with a lead attached somewhere to it 23.

Shot noise. We evaluate now the cross correlations $S(t)$ of the currents by expanding in powers of $V$, see (2). The first non-vanishing contribution is of order $V_{1} V_{2}$. Under the same assumptions as before, we calculate the spectral density of the noise, $S(\omega)=\int d t e^{i \omega t} S(t)$. For the zerofrequency noise we obtain

$$
S(0)=-\frac{e^{2} \Gamma^{4}}{2 \pi} \sum_{i, f, s, s^{\prime}} \rho_{i}\left|\left\langle i\left|D_{2, s^{\prime}}^{\dagger} D_{1, s}\right| f\right\rangle\right|^{2}\left[F_{12}+F_{21}\right] .
$$

We compare now to Eq. (3) and note that depending on the sign of $\mu_{1}-\mu_{2}$ either $F_{12}$ or $F_{21}$ vanish (at $T=0$ ). Therefore, in the cotunneling regime the noise assumes 
its Poissonian value, $S(0)=-e|I|$, and we see that the Fano factor (noise-to-current ratio) is universal and the current and its cross-correlations contain the same information. [When we allow for resonances of the type discussed above, this is no longer the case [23].]

For finite frequencies in the regime $\left|\mu_{1}-\mu_{2}\right|>J$ we obtain after lengthy calculations,

$$
\begin{aligned}
& S(\omega)=\left(e \pi \nu_{t} \Gamma^{2}\right)^{2}\left[X_{\omega}+X_{-\omega}^{*}\right], \\
& \operatorname{Im} X_{\omega}=\frac{C(\varphi)}{2 \omega}\left[\theta\left(\mu_{1}-\omega\right)-\theta\left(\mu_{2}-\omega\right)\right], \\
& \operatorname{Re} X_{\omega}=\frac{C(\varphi)}{2 \pi \omega} \operatorname{sign}\left(\mu_{1}-\mu_{2}+\omega\right) \ln \left|\frac{\left(\mu_{1}+\omega\right)\left(\mu_{2}-\omega\right)}{\mu_{1} \mu_{2}}\right| \\
& -\frac{1}{2 \pi \omega}\left[\theta\left(\omega-\mu_{1}\right) \ln \left|\frac{\mu_{2}-\omega}{\mu_{2}}\right|+\theta\left(\omega-\mu_{2}\right) \ln \left|\frac{\mu_{1}-\omega}{\mu_{1}}\right|\right] .
\end{aligned}
$$

[9] J. M. Kikkawa, D. D. Awschalom, Phys. Rev. Lett. 80,

(10) [11] Note that e.g. a spin singlet in a Cooper pair (in a super-

The real part of $S(\omega)$ is even in $\omega$, while the imaginary part is odd. A remarkable feature here is that the noise acquires an imaginary (i.e. odd-frequency) part for finite frequencies, in contrast to single-barrier junctions, where $\operatorname{Im} S(\omega)$ always vanishes since we have $\delta I_{1}=-\delta I_{2}$ for all times. In double-barrier junctions considered here we find that at small enough bias $\Delta \mu=\mu_{1}-\mu_{2} \ll \mu=$ $\left(\mu_{1}+\mu_{2}\right) / 2$, the odd part, $\operatorname{Im} S(\omega)$, given in (9) exhibits two narrow peaks at $\omega= \pm \mu$, which in real time lead to slowly decaying oscillations,

$$
S_{\text {odd }}(t)=e^{2} \pi \nu_{t}^{2} \Gamma^{4} C(\varphi) \frac{\sin (\Delta \mu t / 2)}{\mu t} \sin (\mu t) .
$$

These oscillations again depend on the phase-coherence factor $C$ with the same properties as discussed before. These oscillations can be interpreted as a temporary build-up of a charge-imbalance on the DD during an uncertainty time $\sim \mu^{-1}$, which results from cotunneling of electrons and an associated time delay between out- and ingoing currents.

Finally, allowing currents to flow from feeding leads via DD into leads 1,2 we can arrange for scattering of unentangled electrons (as considered previously in noise [27]) but now also of entangled ones. In the latter case we get a non-trivial Fano factor [28] due to antibunching (triplets) and bunching (singlet) effects in the noise [10].

Acknowledgements. We would like to thank R. Blick, C. Bruder, G. Burkard, and D. DiVincenzo for discussions. This work has been supported by the Swiss NSF.

[1] A. Einstein, B. Podolsky, N. Rosen, Phys. Rev.47, 777 (1935).

[2] C. H. Bennett and G. Brassard, in Proceedings of the IEEE International Conference on Computers, Systems and Signal Processing, Bangalore, India (IEEE, New York, 1984), p. 175. 4313 (1998); J.A. Gupta et al., Phys. Rev. B59, R10421 (1999).

[10] D.P. DiVincenzo, D. Loss, cond-mat/9901137. conductor)is also entangled but here the singlet consists of orbitally delocalized electrons.

[3] A. Aspect, J. Dalibard, G. Roger, Phys. Rev. Lett. 49, 1804 (1982); W. Tittel et al., Phys. Rev. Lett. 81, 3563 (1998).

[4] A. Zeilinger, in Physics World, March 1998.

[5] A. Steane, Rept. Prog. Phys. 61, 117-173 (1998).

[6] D. Loss, D. P. DiVincenzo, Phys. Rev. A 57, 120 (1998).

[7] G. Burkard, D. Loss, D. P. DiVincenzo, Phys. Rev. B 59 2070 (1999).

[8] Spin-spin coupling can also be achieved on a long distance scale by using a cavity-QED, see A. Imamoglu et al., quant-ph/9904096.

12] R. H. Blick et al., Phys. Rev. Lett. 80, 4032 (1998).

[13] T. H. Oosterkamp et al., Nature 395 873, (1998).

[14] D. V. Averin, Yu. V. Nazarov, in Single Charge Tunneling, eds. H. Grabert and M.H. Devoret, NATO ASI Series B: Physics Vol. 294, Plenum Press, New York, 1992.

[15] We can express $E_{s / t}$ in terms of the single-particle energy $\epsilon$ and of the Coulomb charging energy $E_{c}=e^{2} / 2 C$ (with $C$ being the dot capacitance), $E_{s / t}=2 \epsilon+2 E_{C} \mp J / 2$.

[16] G. D. Mahan, Many Particle Physics, 2nd Ed. (Plenum, New York, 1993).

[17] For experiments on single dots in the cotunneling regime see, D.C. Glattli et al., Z. Phys. B 85, 375 (1991).

[18] Note that our cotunneling regime ensures that the current and noise measure the equilibrium state of the DD.

[19] We note that a recent experiment [20 demonstrated that a single quantum dot which is part of an AB-loop and which operates in the $\mathrm{CB}$ regime supports phase-coherent single-particle transport with AB oscillations.

[20] A. Yacoby et al., Phys. Rev. Lett. 74, 4047 (1995).

[21] One can show that contributions to the current due to virtually double-occupied dots are of relative order $\left(\mu_{1,2} / U\right)^{2} \ll 1$ for $U>\mu_{1,2}$ when compared to the leading term (5); this holds close to resonance, i.e. when $\mu_{1,2}$ are close to the ground state energy $E_{s}$.

[22] Elastic cotunneling through a DD was studied in a "golden rule" approach by H. Akera, Phys. Rev. B47, 6835 (1993).

[23] E. Sukhorukov, D. Loss, to be published.

[24] Note that the AB effect is not suppressed by this inelastic cotunneling, since the entire cotunneling process involving also leads is elastic: the initial and final states of the entire system have the same energy.

[25] We note that this observation agrees with similar findings for two-terminal AB set-ups discussed by M. Büttiker, Phys. Rev. Lett. 68, 843 (1992).

[26] D. Loss, P. Goldbart, Phys. Rev. B 45 (1992) 13544.

[27] M. Büttiker, Phys. Rev. B46, 12485 (1992); T. Martin, R. Landauer, Phys. Rev. B45, 1742 (1992); R. C. Liu et al., Nature 391, 263 (1998); M. Henny et al., Science 284, 296 (1999); W. D. Oliver et al., ibid. 299.

[28] G. Burkard, D. Loss, E. Sukhorukov, cond-mat/9906071. 